\title{
openheart Use of bioresorbable vascular scaffold: a meta-analysis of patients with coronary artery disease
}

\author{
Mohamed Farag, ${ }^{1,2}$ Nikolaos Spinthakis, ${ }^{1}$ Diana A Gorog, ${ }^{1,2,3}$ Abhiram Prasad, ${ }^{4}$ \\ Keith Sullivan, ${ }^{2}$ Zaki Akhtar, ${ }^{1}$ Neville Kukreja, ${ }^{1}$ Manivannan Srinivasan ${ }^{1}$
}

To cite: Farag M, Spinthakis N, Gorog DA, et al. Use of bioresorbable vascular scaffold: a metaanalysis of patients with coronary artery disease. Open Heart 2016;3:e000462. doi:10.1136/openhrt-2016000462

- Additional material is available. To view please visit the journal (http://dx.doi.org/ 10.1136/openhrt-2016000462).

MF and NS contributed equally to this manuscript.

Received 24 April 2016 Revised 4 July 2016 Accepted 24 July 2016
CrossMark

For numbered affiliations see end of article.

\section{Correspondence to} Dr Manivannan Srinivasan; manivannan.srinivasan@nhs. net

\section{ABSTRACT}

Background: Differences in outcomes between bioresorbable vascular scaffold (BVS) systems and drug-eluting metal stents (DES) have not been fully evaluated. We aimed to compare clinical and angiographic outcomes in randomised studies of patients with coronary artery disease (CAD), with a secondary analysis performed among registry studies.

Methods: A meta-analysis comparing outcomes between BVS and DES in patients with CAD. Overall estimates of treatment effect were calculated with random-effects model and fixed-effects model.

Results: In 6 randomised trials (3818 patients), BVS increased the risk of subacute stent thrombosis (ST) over and above DES (OR 2.14; Cl 1.01 to 4.53; $p=0.05)$, with a trend towards an increase in the risk of myocardial infarction (MI) (125 events in those assigned to BVS and 50 to DES; OR 1.36; $\mathrm{Cl} 0.97$ to $1.91 ; p=0.07$ ). The risk of in-device late lumen loss (LLL) was higher with BVS than DES (mean difference $0.08 \mathrm{~mm} ; \mathrm{Cl} 0.03$ to $0.13 ; p=0.004$ ). There was no difference in the risk of death or target vessel revascularisation (TVR) between the two devices. In 6 registry studies (1845 patients), there was no difference in the risk of death, MI, TVR or subacute ST between the two stents. Final BVS dilation pressures were higher in registry than in randomised studies $(18.7 \pm 4.6$ vs $15.2 \pm 3.3 \mathrm{~atm} ; p<0.001)$.

Conclusions: Patients treated with BVS had an increased risk of subacute ST and slightly higher LLL compared with those with DES, but this might be related to inadequate implantation techniques, in particular device underexpansion.

\section{INTRODUCTION}

Despite latest-generation drug-eluting metal stents (DES) demonstrating improved safety and efficacy compared with first-generation implants, ${ }^{1}$ there remains the need for better devices for percutaneous coronary intervention (PCI). Recent clinical studies using the first commercially available bioresorbable vascular scaffold (BVS) systems have reported promising results. Unlike DES, BVS potentially allow restoration of anatomy and

\section{KEY QUESTIONS}

What is already known about this subject?

- Recent clinical studies using the first commercially available bioresorbable vascular scaffold systems have reported promising results. However, the rate of subacute stent thrombosis was observed to be higher than expected after implantation of these devices, possibly due to suboptimal device expansion and insufficient intracoronary imaging guidance.

What does this study add?

- The aim of this study was to evaluate the efficacy and safety of bioresorbable vascular scaffold systems, compared with drug-eluting metal stents, using the data available from randomised and observational studies, with a focus on stent implantation techniques.

How might this impact on clinical practice?

- When using bioresorbable vascular scaffold systems, every effort should be made to select suitable patients, with careful lesion preparation, and with intervention guided by adequate intravascular device optimisation.

vascular function. This novel technology offers the promise of eliminating late stent failure related to thrombosis and neoatherosclerosis that is associated with the presence of a permanent metallic foreign body. However, in early registry studies, BVS use was associated with higher rates of stent thrombosis (ST) in the first 6 months, ${ }^{2-4}$ possibly due to suboptimal device expansion and insufficient intracoronary imaging guidance.

The currently available data from individual randomised trials and observational studies suggest that clinical outcomes are otherwise similar between patients treated with DES or BVS, ${ }^{5-16}$ though most studies are limited by a small sample size. The aim of this meta-analysis was to evaluate the efficacy and safety of BVS, compared with DES, 
using the data available from randomised trials, with a focus on stent implantation techniques. We also performed an analysis of data from observational studies in order to determine outcomes in the 'real-world' setting.

\section{METHODS}

\section{Study objectives and design}

The outcomes of interest were death, myocardial infarction (MI), target vessel revascularisation (TVR), ST and in-device late lumen loss (LLL) at angiographic follow-up, defined according to the study protocols. We also performed a secondary analysis for death, MI, TVR and ST for registry studies. The study was designed according to the PRISMA (Preferred Reporting Items for Systematic Reviews and Meta-analyses) statement and the Meta-Analysis of Observational Studies in Epidemiology (MOOSE) work groups. ${ }^{17} 18$

\section{Study search strategy}

Using MEDLINE, Embase, Scopus and the Cochrane Library, we performed searches of articles published until December 2015, without language restrictions. Eligible studies were identified using various combinations of the terms: absorb, bioresorbable, drug-eluting stent, percutaneous, coronary, MI, angina, angioplasty and intervention in the abstract or title. Reference lists of the retrieved articles were reviewed to identify eligible studies. Online oral presentations and expert slide presentations were also examined. Two reviewers independently reviewed all titles, or titles and abstracts from the search results to identify articles according to fulfilment of inclusion criteria. Selected trials were compared, and disagreement was resolved by team discussion and consensus. Studies were excluded from the meta-analysis if they included the use of bare-metal stent, reported duplicate or incomplete interim data, had small sample size of $\leq 50$ patients or short follow-up of $<3$ months or were single-arm studies.

\section{Data extraction}

Data extraction was carried out independently and in duplicate by the study investigators. Results of data extraction were then compared, and discrepancies resolved by consensus. If results were incomplete or unclear, the study authors were contacted. Articles selected for the final review were checked to avoid inclusion of data published in duplicate. Data were collated from each study regarding baseline characteristics, including study design, device type, sample characteristics, ACC/AHA lesion classification, glycoprotein IIb/ IIIa inhibitors (GPI) use, use of intracoronary imaging, predilation and postdilation pressures, duration of dual antiplatelet therapy and procedural and clinical outcomes at the end of follow-up. If TVR was not reported, we used target lesion revascularisation instead. As for ST, we pooled outcomes for any ST, including definite, probable and possible ST. All outcomes were defined according to the original study's protocol definition. ST was defined according to the Academic Research Consortium criteria $^{19}$ in all included studies. When a study reported follow-up at 1 and 2 years, outcomes for the longer time periods were abstracted.

\section{Statistical analysis}

Outcomes are presented as OR with 95\% CI. Pooled OR was calculated for categorical and continuous variables using a random-effects model by the method of DerSimonian and Laird. ${ }^{20}$ Heterogeneity was assessed using $\chi^{2}$ and $\mathrm{I}^{2}$ tests. We also used the Mantel-Haenszel fixed-effects model, when no heterogeneity was observed (ie, $\mathrm{I}^{2}=0 \%$ ). All analyses were performed with the intention-to-treat principle. In sensitivity analysis, we included only registry studies which directly compared BVS with DES in coronary artery disease (CAD). We did meta-regression to examine the relationship between the log-transformed OR of the effect of BVS on ST risk and the log-transformed OR of the effect of BVS on mortality, in identified randomised trials. We used the unpaired two-sample mean comparison Student's t-test to compare weighted means of different populations, in particular those related to stent technical parameters (ie, BVS final dilation pressure).

Publication bias was minimised by a comprehensive and inclusive literature search. In addition, a graphical display (funnel plot) of the size of the treatment effect against the precision of the trial $(1 / \mathrm{SE})$ was used to investigate publication bias. All tests were two-sided, and statistical significance was fixed at 0.05 level. Analysis was carried out using Review Manager Software (RevMan V.5.3) and Stata V.11.2 (StataCorp, College Station, Texas, USA).

\section{RESULTS}

Six randomised trials involving 3818 patients were identified, which directly compared the clinical outcomes of BVS versus DES in CAD (see online supplementary figure S1). ${ }^{5-10}$ Six registry studies ${ }^{11-16}$ involving 1845 patients that compared clinical outcomes of BVS against DES were also included. Of these, three performed propensity score matched-sample analyses. ${ }^{11} 1314$ The methodological quality of included studies is described in online supplementary tables S1 and S2. The primary outcomes of included studies are listed in online supplementary tables S3 and S4. There was no evidence of publication bias having a significant effect on the results (see online supplementary figure S2).

The characteristics of randomised trials are listed in table 1. Greater than three-quarters of patients were male with a mean age of $62 \pm 4$ years, $28.4 \%$ patients with diabetes and ACC/AHA lesion class was $\mathrm{B} 2 / \mathrm{C}$ in $66.2 \%$. All trials included patients with acute coronary syndromes (table 1). The median follow-up was 12 months. Patients randomised to receive BVS $(n=2337)$ were treated with the Absorb everolimus-eluting scaffold 
Table 1 Baseline patient and lesion characteristics of randomised trials

\begin{tabular}{|c|c|c|c|c|c|c|}
\hline & $\begin{array}{l}\text { ABSORB II, } \\
2015\end{array}$ & $\begin{array}{l}\text { ABSORB III, } \\
2015\end{array}$ & $\begin{array}{l}\text { ABSORB } \\
\text { CHINA, } 2015 \\
\end{array}$ & $\begin{array}{l}\text { ABSORB } \\
\text { JAPAN, } 2015\end{array}$ & $\begin{array}{l}\text { EVERBIO II, } \\
2015\end{array}$ & $\begin{array}{l}\text { ABSORB STEMI } \\
\text { TROFI II ST, } 2016\end{array}$ \\
\hline Patients (n) & 501 & 2008 & 480 & 400 & 238 & 191 \\
\hline BVS (n) & 335 & 1322 & 241 & 266 & 78 & 95 \\
\hline DES (n) & 166 & 686 & 239 & 134 & 160 & 96 \\
\hline Male (\%) & 77 & 70 & 72 & 77 & 79 & 82 \\
\hline Mean age (year) & 61 & 63 & 57 & 67 & 65 & 59 \\
\hline Diabetes (\%) & 24 & 31 & 24 & 36 & 23 & 16 \\
\hline Follow-up (months) & 24 & 12 & 12 & 12 & 9 & 6 \\
\hline \multicolumn{7}{|l|}{ GPI use (\%) } \\
\hline BVS & NR & 10.1 & NR & NR & NR & 40 \\
\hline DES & NR & 12.4 & NR & NR & NR & 38.5 \\
\hline $\begin{array}{l}\text { DAPT duration } \\
\text { (months) }\end{array}$ & Up to 24 & 12 & 12 & 12 & 6 & 12 \\
\hline Device type & BVS, EES & BVS, EES & BVS, EES & BVS, EES & $\begin{array}{l}\text { BVS, EES, } \\
\text { BES }\end{array}$ & BVS, EES \\
\hline \multicolumn{7}{|c|}{ Intracoronary imaging used (OCT/IVUS \%) } \\
\hline BVS & 100 & 11.2 & 0.4 & 100 & NR & 0 \\
\hline DES & 100 & 10.8 & 0.4 & 100 & NR & 0 \\
\hline \multicolumn{7}{|c|}{ ACC/AHA lesion class (\%) } \\
\hline$A$ & 1.1 & NR & 9.5 & 3.8 & 24.9 & NR \\
\hline B1 & 52.1 & NR & 21.7 & 20.1 & 44 & NR \\
\hline B2 & 45.3 & $69.9^{\star}$ & 49 & 53.8 & 15 & NR \\
\hline C & 1.4 & & 24.5 & 22 & 16 & NR \\
\hline \multicolumn{7}{|c|}{ Device predilation (\%) } \\
\hline BVS & 100 & 100 & 99.6 & 100 & NR & 55.8 \\
\hline DES & 99 & 100 & 98 & 100 & NR & 51 \\
\hline \multicolumn{7}{|c|}{ Device postdilation (\%) } \\
\hline BVS & 61 & 65.5 & 63 & 82.2 & 34 & 50.5 \\
\hline DES & 59 & 51.2 & 54.4 & 77.4 & 31 & 25.5 \\
\hline \multicolumn{7}{|c|}{ Expected maximum diameter of postdilation balloon (mm) } \\
\hline BVS & 3.29 & 3.18 & 3.3 & 3.34 & NR & 3.51 \\
\hline DES & 3.35 & 3.12 & 3.2 & 3.31 & NR & 3.29 \\
\hline \multicolumn{7}{|c|}{ Final dilation pressure (atm) } \\
\hline BVS & 14.2 & 15.4 & 16.8 & 14.7 & 13.6 & 15.8 \\
\hline DES & 15 & 15.4 & 16.9 & 15.1 & 14.2 & 18.6 \\
\hline \multicolumn{7}{|c|}{ Clinical presentation (\%) } \\
\hline STEMI & 0 & 0 & 0 & 0 & 9.6 & 100 \\
\hline Non-STEMI & 0 & 0 & 0 & 0 & 21 & 0 \\
\hline Unstable angina & 20.9 & 26 & 64.4 & 12 & 8.4 & 0 \\
\hline Stable angina & 63.8 & 58.4 & 19.5 & 64.5 & 48.3 & 0 \\
\hline Silent ischaemia & 12.1 & 10 & 4.6 & 23.5 & 12.6 & 0 \\
\hline
\end{tabular}

Values are means or (\%).

${ }^{*} \mathrm{~B} 2+\mathrm{C}$ lesions.

BES, biolimus-eluting stent; BVS, bioresorbable vascular scaffold; DAPT, dual antiplatelet therapy; DES, drug-eluting stent; EES, everolimus eluting stent; GPI, glycoprotein IIb/IIla inhibitors; IVUS, intravascular ultrasound; NR, not reported; OCT, optical coherence tomography; STEMI, ST-elevation myocardial infarction.

(Abbott Vascular, Santa Clara, California, USA). BVS postdilation was performed in $64.8 \%$ of patients up to a mean maximum pressure of $15.2 \pm 3.3$ atm. Intravascular imaging (OCT/IVUS) was used to guide BVS expansion in $33.2 \%$ of cases. Patients randomised to receive DES $(n=1401)$ were treated with either an everolimus-eluting cobalt-chromium stent (Xience Prime, Xience V, or Xience Xpedition; Abbott Vascular, Santa Clara, California, USA), an everolimus-eluting platinum-chromium stent (Promus Element; Boston Scientific, Natick, Massachusetts, USA) or a biolimus-eluting stainless-steel stent (Biomatrix Flex; Biosensors, Europe, SA). Four of the clinical trials' primary end points were based on angiographic criteria. ${ }^{5-8}$ In one trial, a 2-year interim clinical outcomes report was only available online, and these results were used. ${ }^{9}$ All interventions were performed according to standard of care, including optimisation of stent deployment or use of intravascular imaging techniques, according to trial protocols. All patients were prescribed long-term aspirin, whereas the use of dual antiplatelet therapy varied between 6 and 12 months. All trials reported the adherence to dual 
antiplatelet therapy to be $>80 \%$ up to the longest follow-up period available.

In the randomised trials, there was no difference in the risk of death (OR 1.29; CI 0.43 to 3.83 ; $\mathrm{I}^{2}=23 \%$; $\mathrm{p}=0.65$ ) (figure $1 \mathrm{~A}$ ) or TVR (OR 1.15; CI 0.83 to 1.59 ; $\mathrm{I}^{2}=0 \% ; \mathrm{p}=0.40(\mathrm{p}=0.38$ by fixed-effect model $)$ ) (figure 1B) between the two devices. Compared with DES, patients treated with BVS showed a trend towards an increase in the risk of MI (125 events in those assigned to BVS and 50 to DES; OR 1.36; CI 0.97 to $1.91 ; \mathrm{I}^{2}=0 \%$; $\mathrm{p}=0.07$ ) ( $\mathrm{p}=0.06$ by fixed-effect model)) (figure 1C). Patients treated with BVS had a higher rate of any ST (definite, probable or possible ST; with the majority of events occurring within 30 days; $n=39$ ) compared with those treated with DES (OR 2.14; CI 1.01 to $4.53 ; \mathrm{I}^{2}=0 \%$; $\mathrm{p}=0.05 \quad(\mathrm{p}=0.03$ by fixed-effect model) $)$ (figure $2 \mathrm{~A})$. Definite ST occurred in $33(0.9 \%)$ patients. There was a trend towards an increase in the risk of definite ST (27 events in those assigned to BVS and 6 to DES; OR 2.11; CI 0.92 to $4.88 ; \mathrm{I}^{2}=0 \%$; $\mathrm{p}=0.08$ ) ( $\mathrm{p}=0.07$ by fixed-effect model)) (figure 2B). There was no correlation between the increase in ST risk with BVS and mortality among the trials reporting at least one ST and death event ( $\mathrm{p}=0.66$; figure 3$)$.
In trials with angiographic surveillance, the median angiographic follow-up was 10.5 months (IQR 7.5-12.5). Coronary lesions treated with BVS had significantly higher in-device LLL at angiographic follow-up than those treated with DES (mean difference between groups $0.08 \mathrm{~mm}$; CI 0.03 to $0.13 ; \mathrm{I}^{2}=52 \% ; \mathrm{p}=0.004$ ) (figure 2C).

The characteristics of registry studies are listed in table 2. Greater than three-quarters of patients enrolled were male with a mean age of $62 \pm 3$ years, $18.2 \%$ patients with diabetes and ACC/AHA lesion class was B2/C in 84.7\%. All studies included patients with acute coronary syndromes (table 2). The median follow-up was 8 months. Patients receiving BVS $(n=785)$ were treated with the Absorb everolimus-eluting scaffold. BVS postdilation was performed in $60.0 \%$ of all patients up to a mean maximum pressure of $18.7 \pm 4.6 \mathrm{~atm}$. Intravascular imaging (OCT/ IVUS) was used to guide BVS expansion in $63.7 \%$ of cases. Patients receiving DES $(\mathrm{n}=1060)$ were treated with an everolimus-eluting cobalt-chromium stent (Xience Prime, Xience Pro, Xience V, or Xience Xpedition; Abbott Vascular, Santa Clara, California, USA), an everolimus-eluting platinum-chromium stent (Promus, Promus Element, Premier; Boston Scientific, Natick,

\begin{tabular}{|c|c|c|c|c|c|c|c|c|c|c|c|}
\hline \multirow[b]{2}{*}{ Study or Subgroup } & \multicolumn{2}{|c|}{ BVS } & \multicolumn{2}{|c|}{ DES } & \multicolumn{3}{|c|}{ Odds Ratio } & \multirow{2}{*}{\multicolumn{4}{|c|}{$\begin{array}{c}\text { Odds Ratio } \\
\text { M-H, Random, } 95 \% \mathrm{Cl} \\
\end{array}$}} \\
\hline & Events & Total & Events & Total & Weight & M-H, Random, $95 \% \mathrm{CI}$ & Year & & & & \\
\hline EVERBIO II & 1 & 78 & 3 & 160 & $17.9 \%$ & $0.68[0.07,6.64]$ & 2014 & $\leftarrow$ & $=-$ & & \\
\hline ABSORB JAPAN & 2 & 265 & 0 & 133 & $11.1 \%$ & $2.53[0.12,53.14]$ & 2015 & & & & \\
\hline ABSORB CHINA & 0 & 238 & 5 & 237 & $12.1 \%$ & $0.09[0.00,1.61]$ & 2015 & $\leftarrow$ & & & \\
\hline ABSORB II & 4 & 335 & 1 & 166 & $18.9 \%$ & $1.99[0.22,17.98]$ & 2015 & & & & \\
\hline ABSORB STEMI TROFI II ST & 0 & 95 & 0 & 96 & & Not estimable & 2015 & & & & \\
\hline ABSORB III & 15 & 1313 & 3 & 677 & $40.0 \%$ & $2.60[0.75,9.00]$ & 2015 & & & - & \\
\hline Total $(95 \% \mathrm{Cl})$ & & 2324 & & 1469 & $100.0 \%$ & $1.29[0.43,3.83]$ & & & & & \\
\hline Total events & 22 & & 12 & & & & & & & & \\
\hline \multicolumn{8}{|c|}{$\begin{array}{l}\text { Heterogeneity: } \text { Tau }^{2}=0.37 ; \mathrm{Chi}^{2}=5.22, \mathrm{df}=4(\mathrm{P}=0.27) ; \mathrm{F}^{2}=23 \% \\
\text { Test for overall effect: } Z=0.46(P=0.65)\end{array}$} & $\begin{array}{cc}\vdash & 1 \\
0.1 & 0.2\end{array}$ & $\begin{array}{c}0.5 \\
\text { Favours BVS }\end{array}$ & $\begin{array}{l}\frac{1}{2} \\
\text { Favours DES }\end{array}$ & $\frac{1}{5}$ \\
\hline
\end{tabular}

B

\begin{tabular}{|c|c|c|c|c|c|c|c|c|c|c|c|c|}
\hline \multirow[b]{2}{*}{ Study or Subgroup } & \multicolumn{2}{|c|}{ BVS } & \multicolumn{2}{|c|}{ DES } & \multicolumn{3}{|c|}{ Odds Ratio } & \multirow{2}{*}{\multicolumn{5}{|c|}{$\begin{array}{c}\text { Odds Ratio } \\
\text { M-H, Random, } 95 \% \mathrm{Cl}\end{array}$}} \\
\hline & Events & Total & Events & Total & Weight & M-H, Random, 95\% CI & Year & & & & & \\
\hline EVERBIO II & 11 & 78 & 22 & 160 & $17.0 \%$ & $1.03[0.47,2.25]$ & 2014 & & & & & \\
\hline ABSORB II & 9 & 335 & 3 & 166 & $6.0 \%$ & $1.50[0.40,5.62]$ & 2015 & & & & & \\
\hline ABSORB JAFAN & 13 & 265 & 6 & 133 & $10.6 \%$ & $1.09[0.41,2.94]$ & 2015 & & & & & \\
\hline ABSORB CHINA & 9 & 238 & 12 & 237 & $13.3 \%$ & $0.74[0.30,1.78]$ & 2015 & & & & & \\
\hline ABSORB III & 69 & 1313 & 28 & 677 & $51.4 \%$ & $1.29[0.82,2.01]$ & 2015 & & & & & \\
\hline ABSORB STEMI TROFI II ST & 2 & 95 & 1 & 96 & $1.8 \%$ & $2.04[0.18,22.92]$ & 2015 & & & & & \\
\hline Total $(95 \% \mathrm{Cl})$ & & 2324 & & 1469 & $100.0 \%$ & $1.15[0.83,1.59]$ & & & & & & \\
\hline Total events & 113 & & 72 & & & & & & & & & \\
\hline $\begin{array}{l}\text { Heterogeneity: } \text { Tau }^{2}=0.00 ; 0 \\
\text { Test for overall effect: } Z=0.8\end{array}$ & $\begin{array}{l}i^{2}=1.67, \\
P P=0.40\end{array}$ & $d f=50$ & $(\mathrm{P}=0.89)$ & $P^{2}=0$ & & & & $\begin{array}{ll}0.1 & 0.2\end{array}$ & $\begin{array}{c}0.5 \\
\text { Favours BVS }\end{array}$ & $\begin{array}{c}2 \\
\text { Favours DES }\end{array}$ & 5 & 10 \\
\hline
\end{tabular}

Test for overall effect using fixed-effect model: $Z=0.88(P=0.38)$

C

\begin{tabular}{|c|c|c|c|c|c|c|c|c|c|c|c|}
\hline \multirow[b]{2}{*}{ Study or Subgroup } & \multicolumn{2}{|c|}{ BVS } & \multicolumn{2}{|c|}{ DES } & \multirow[b]{2}{*}{ Weight } & \multicolumn{2}{|l|}{ Odds Ratio } & \multirow{2}{*}{\multicolumn{4}{|c|}{$\begin{array}{c}\text { Odds Ratio } \\
\text { M-H, Random, } 95 \% \mathrm{Cl} \\
\end{array}$}} \\
\hline & Events & Total & Events & Total & & M-H, Random, $95 \% \mathrm{CI}$ & Year & & & & \\
\hline EVERBIO II & 1 & 78 & 1 & 160 & $1.5 \%$ & $2.06[0.13,33.46]$ & 2014 & & & & \\
\hline ABSORB JAPAN & 9 & 265 & 3 & 133 & $6.5 \%$ & $1.52[0.41,5.72]$ & 2015 & & & & \\
\hline ABSORB CHINA & 5 & 238 & 4 & 237 & $6.5 \%$ & $1.25[0.33,4.71]$ & 2015 & & & & \\
\hline ABSORB II & 19 & 335 & 4 & 166 & $9.5 \%$ & $2.44[0.81,7.28]$ & 2015 & & - & - & \\
\hline ABSORB STEMI TROFI II ST & 1 & 95 & 0 & 96 & $1.1 \%$ & $3.06[0.12,76.15]$ & 2015 & & & & \\
\hline ABSORB III & 90 & 1313 & 38 & 677 & $74.8 \%$ & $1.24[0.84,1.83]$ & 2015 & & & & \\
\hline Total $(95 \% \mathrm{Cl})$ & & 2324 & & 1469 & $100.0 \%$ & $1.36[0.97,1.91]$ & & & & & \\
\hline Total events & 125 & & 50 & & & & & & & & \\
\hline $\begin{array}{l}\text { Heterogeneity: } T_{a u}=0.00 ; 0 \\
\text { Test for overall effect: } Z=1.70\end{array}$ & $\begin{array}{l}i^{2}=1.69, \\
(P=0.07\end{array}$ & $d f=5$ & $P=0.89)$ & $z_{12}^{2}=0$ & & & & $\begin{array}{ll}0.1 & 1 \\
0.2\end{array}$ & $\begin{array}{c}1 \\
0.5 \\
\text { Favours Bvs }\end{array}$ & $\begin{array}{l}1 \\
1 \\
\text { Favours DES }\end{array}$ & $\frac{1}{5}$ \\
\hline
\end{tabular}

Test for overall effect using fixed-effect model: $\mathrm{Z}=1.87(\mathrm{P}=0.06)$

Figure 1 Death, target vessel revascularisation (TVR) and myocardial infarction (MI) with bioresorbable vascular scaffold (BVS) versus drug-eluting stent (DES) in randomised trials in coronary artery disease. (A) Death, (B) TVR and (C) MI. 


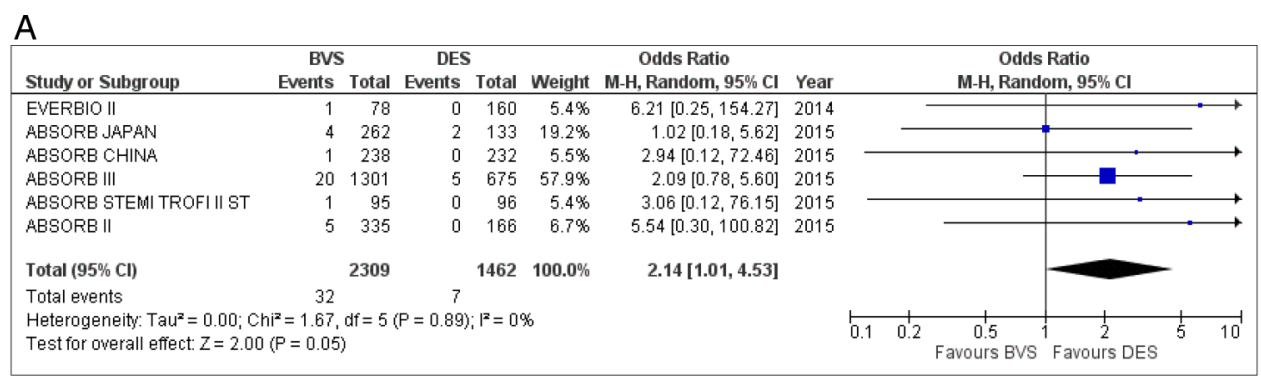

Test for overall effect using Fixed Effect: $\mathrm{Z}=2.14(\mathrm{P}=0.03)$

B

\begin{tabular}{|c|c|c|c|c|c|c|c|c|c|c|c|}
\hline \multirow[b]{2}{*}{ Study or Subgroup } & \multicolumn{2}{|c|}{ BVS } & \multicolumn{2}{|c|}{ DES } & \multicolumn{3}{|c|}{ Odds Ratio } & \multirow{2}{*}{\multicolumn{4}{|c|}{$\begin{array}{c}\text { Odds Ratio } \\
\text { M-H, Random, } 95 \% \mathrm{Cl}\end{array}$}} \\
\hline & Events & Total & Events & Total & Weight & M-H, Random, $95 \% \mathrm{Cl}$ & Year & & & & \\
\hline EVEREIO II & 0 & 78 & 0 & 160 & & Not estimable & 2014 & & & & \\
\hline ABSORB III & 18 & 1301 & 5 & 675 & $70.6 \%$ & $1.88[0.69,5.09]$ & 2015 & & & & \\
\hline ABSORB STEMI TROFI II ST & 1 & 95 & 0 & 96 & $6.8 \%$ & $3.06[0.12,76.15]$ & 2015 & & & & \\
\hline ABSORB CHINA & 0 & 238 & 0 & 232 & & Not estimable & 2015 & & & & \\
\hline ABSORB II & 4 & 335 & 0 & 166 & $8.2 \%$ & $4.52[0.24,84.46]$ & 2015 & & & & \\
\hline ABSORB JAPAN & 4 & 262 & 1 & 133 & $14.4 \%$ & $2.05[0.23,18.49]$ & 2015 & & & & \\
\hline Total $(95 \% \mathrm{Cl})$ & & 2309 & & 1462 & $100.0 \%$ & $2.11[0.92,4.88]$ & & & & & \\
\hline Total events & 27 & & 6 & & & & & & & & \\
\hline $\begin{array}{l}\text { Heterogeneity: } \mathrm{Tau}^{\bar{z}}=0.00 ; 0 \\
\text { Test for overall effect: } Z=1.7\end{array}$ & $\begin{array}{l}F^{2}=0.37, \\
(P=0.08)\end{array}$ & $d f=3$ & $P=0.95)$ & $; I^{2}=09$ & & & & $0.1 \quad 0.2$ & $\begin{array}{c}0.5 \\
\text { Favours BVs }\end{array}$ & $\frac{1}{2}$ Favours DES & 10 \\
\hline
\end{tabular}

Test for overall effect using Fixed Effect: $\mathrm{Z}=1.82(\mathrm{P}=0.07)$

C

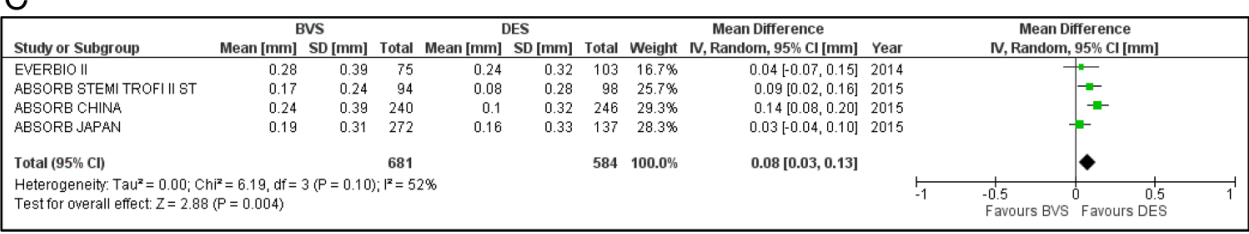

Figure 2 Any and definite stent thrombosis (ST) and in-device late lumen loss (LLL) with bioresorbable vascular scaffold (BVS) versus drug-eluting stent (DES) in randomised trials in coronary artery disease. (A) any ST, (B) definite ST and (C) in-device LLL.

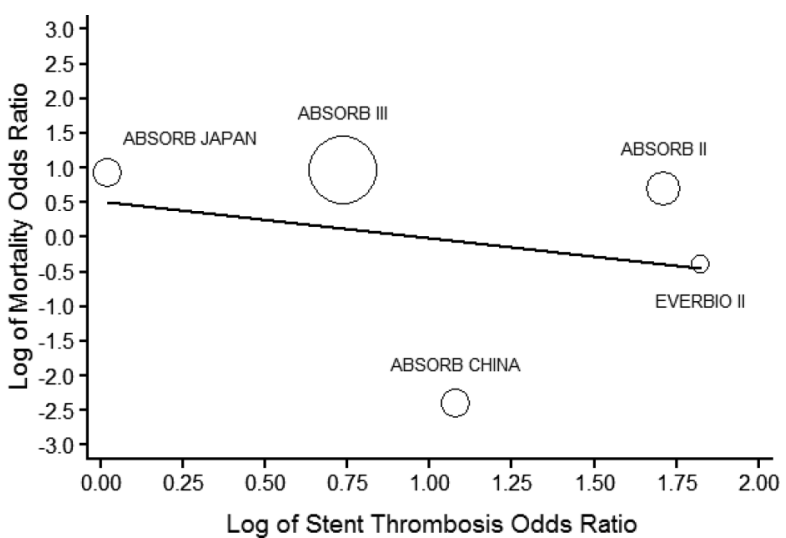

Figure 3 Relationship between stent thrombosis (ST) and mortality, restricted to trials with at least one ST and death event. Slope of the regression line $-0.53, p=0.6556$.

Massachusetts, USA), a biolimus-eluting stainless-steel stent (N-BES, Nobori; Terumo, Tokyo, Japan) or a zotarolimus-eluting cobalt-chromium stent (Medtronic Vascular, Santa Rosa, California, USA). All patients were prescribed long-term aspirin and dual antiplatelet therapy was given for 12 months. Only one study reported the adherence to prescribed dual antiplatelet therapy in the BVS arm up to 12 months, to be $100 \% .^{11}$
In registry studies, there was no difference in the risk of death (OR 0.64; CI 0.27 to $1.48 ; \mathrm{I}^{2}=0 \% ; \mathrm{p}=0.30$ ( $\mathrm{p}=0.22$ by fixed-effect model) ) (figure 4A), TVR (OR $0.85 ;$ CI 0.49 to $1.48 ; \mathrm{I}^{2}=0 \% ; \mathrm{p}=0.57 \quad(\mathrm{p}=0.54$ by fixed-effect model)) (figure 4B), MI (OR 1.37; CI 0.77 to $\left.2.43 ; \mathrm{I}^{2}=0 \% ; \mathrm{p}=0.28\right) \quad(\mathrm{p}=0.30$ by fixed-effect model $)$ ) (figure $4 \mathrm{C}$ ), any ST (OR 1.21; CI 0.58 to $2.51 ; \mathrm{I}^{2}=0 \%$; $\mathrm{p}=0.61 \quad(\mathrm{p}=0.35$ by fixed-effect model $)$ ) (figure $5 \mathrm{~A}$ ) or definite ST (OR 1.27; CI 0.58 to 2.82; $\mathrm{I}^{2}=0 \%$; $\mathrm{p}=0.55$ ) ( $\mathrm{p}=0.65$ by fixed-effect model) ) (figure $5 \mathrm{~B}$ ) between the two devices.

The results observed in all included registry studies were also observed within the three studies that had directly compared BVS with DES (see online supplementary figure S3). Final BVS dilation pressures were higher in registries than in randomised trials $(18.7 \pm 4.6$ vs 15.2 $\pm 3.3 \mathrm{~atm} ; \mathrm{p}<0.001$ ), with a mean diameter of postdilation balloons of $3.4 \pm 0.3$ vs $3.2 \pm 0.4 \mathrm{~mm}$, respectively.

\section{DISCUSSION}

The major findings of this meta-analysis of six randomised trials, involving 3818 patients undergoing PCI, are that patients treated with BVS have an increased risk of subacute ST associated with a trend towards an increased risk of MI, compared with those treated with DES. There was also a greater in-device LLL with BVS. However, this was with no difference in TVR or mortality. A difference 
Table 2 Baseline patient and lesion characteristics of registry studies

\begin{tabular}{|c|c|c|c|c|c|c|}
\hline & Mattesini et $a l^{16}$ & $\begin{array}{l}\text { Gori } \\
\text { et } a l^{15}\end{array}$ & Sato et $a l^{11}$ & $\begin{array}{l}\text { Cortese } \\
\text { et } a l^{12}\end{array}$ & BVS-EXAMINATION ${ }^{13}$ & $\begin{array}{l}\text { Costopoulos } \\
\text { et } a I^{14}\end{array}$ \\
\hline Patients (n) & 73 & 253 & 192 & 563 & 580 & 184 \\
\hline BVS (n) & 35 & 150 & 96 & 122 & 290 & 92 \\
\hline DES (n) & 38 & 103 & 96 & 441 & 290 & 92 \\
\hline Male (\%) & 79 & 72 & 89 & 78 & 80 & 87 \\
\hline $\begin{array}{l}\text { Mean age } \\
\text { (years) }\end{array}$ & 62 & 62 & 66 & $59^{*}$ & 57 & 63 \\
\hline Diabetes (\%) & 31 & 17 & 23 & 17 & 13 & 27 \\
\hline $\begin{array}{l}\text { Follow-up } \\
\text { (months) }\end{array}$ & 8 & 6 & 12 & $8^{*}$ & 12 & 6 \\
\hline \multicolumn{7}{|l|}{ GPI use (\%) } \\
\hline BVS & NR & 38.7 & NR & 34.8 & 67.5 & NR \\
\hline DES & NR & 11.7 & NR & 37.4 & 51.7 & NR \\
\hline $\begin{array}{l}\text { DAPT duration } \\
\text { (months) }\end{array}$ & NR & 12 & 12 & 12 & 12 & 12 \\
\hline Device type & BVS, EES, ZES & BVS, EES & BVS, BES, EES & BVS, EES & BVS, EES & BVS, EES \\
\hline \multicolumn{7}{|c|}{ Intracoronary imaging used (OCT/IVUS \%) } \\
\hline BVS & 100 & NR & 91.7 & 4.4 & NR & 99.8 \\
\hline DES & 100 & NR & 64.6 & 0.4 & NR & 16.8 \\
\hline \multicolumn{7}{|c|}{ ACC/AHA lesion class (\%) } \\
\hline A & 0 & NR & NR & NR & NR & NR \\
\hline B1 & 0 & NR & NR & NR & NR & NR \\
\hline B2 & 31 & NR & $84.1 \dagger$ & NR & NR & $80.8 †$ \\
\hline $\mathrm{C}$ & 69 & NR & & NR & NR & \\
\hline \multicolumn{7}{|c|}{ Device predilation (\%) } \\
\hline BVS & 100 & 100 & 100 & 94.1 & 81 & 97.8 \\
\hline DES & 100 & 100 & 72.1 & 60 & 29 & 75.8 \\
\hline \multicolumn{7}{|c|}{ Device postdilation (\%) } \\
\hline BVS & 100 & 14 & 100 & 94.1 & 36.3 & 99.3 \\
\hline DES & 100 & 14 & 86.4 & 37.9 & 15.2 & 77.4 \\
\hline \multicolumn{7}{|c|}{ Expected maximum diameter of postdilation balloon (mm) } \\
\hline BVS & 3.5 & NR & 3.2 & 3.5 & NR & 3.2 \\
\hline DES & 3.5 & NR & 3.15 & 3.5 & NR & 3.1 \\
\hline \multicolumn{7}{|c|}{ Final dilation pressure (atm) } \\
\hline BVS & 21.3 & 15.3 & 21.1 & $10^{*}$ & NR & 20.9 \\
\hline DES & 17.1 & 12.2 & 18.4 & 14 & NR & 19.0 \\
\hline $\begin{array}{l}\text { Clinical } \\
\text { presentation (\%) }\end{array}$ & & & All-comers & & & \\
\hline STEMI & 0 & 40.7 & & 100 & 100 & $11.4 \ddagger$ \\
\hline Non-STEMI & 0 & 41.8 & & 0 & 0 & \\
\hline $\begin{array}{l}\text { Unstable } \\
\text { angina }\end{array}$ & 4.1 & 17.3 & & 0 & 0 & \\
\hline Stable angina & 95.8 & 0 & & 0 & 0 & 88.6 \\
\hline $\begin{array}{l}\text { Silent } \\
\text { ischaemia }\end{array}$ & 0 & 0 & & 0 & 0 & 0 \\
\hline
\end{tabular}

Values are means unless otherwise specified or (\%).

${ }^{\star}$ Expressed as median.

†B2+C lesions.

$\ddagger$ Acute coronary syndrome.

BES, biolimus-eluting stent; BVS, bioresorbable vascular scaffold; DAPT, dual antiplatelet therapy; EES, everolimus-eluting stent; GPI, glycoprotein Ilb/Illa inhibitors; IVUS, intravascular ultrasound; NR, not reported; OCT, optical coherence tomography; STEMI, ST-elevation myocardial infarction; ZES, zotarolimus-eluting stent.

in ST rates was not detected among the 1845 patients from the 6 registry studies, and rates for death, TVR and MI were similar in BVS and DES treated patients.

In randomised trials, the rate of subacute ST was higher with BVS compared with DES, with an overall rate of $1.39 \%$ in 2309 patients receiving BVS and $0.49 \%$ in 1462 patients receiving DES at 12 months. Although postdilation was more frequently performed in the BVS group (table 1), the final result is considered insufficient for adequate BVS implantation as currently recommended. In randomised trials, BVS postdilation was performed in $64.8 \%$ of cases, with final dilation pressures of 


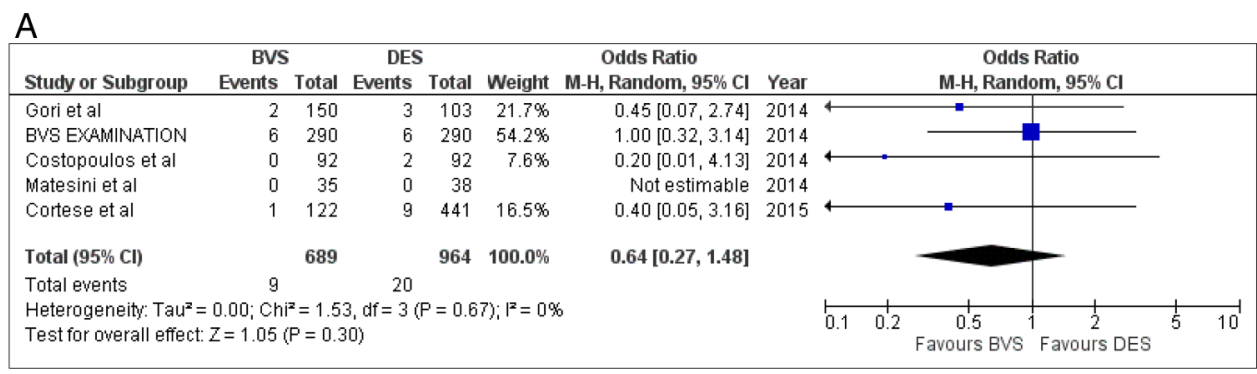

Test for overall effect using Fixed Effect: $\mathrm{Z}=1.22(\mathrm{P}=0.22)$

B

\begin{tabular}{|c|c|c|c|c|c|c|c|c|c|c|c|c|}
\hline \multirow[b]{2}{*}{ Study or Subgroup } & \multicolumn{2}{|c|}{ BVS } & \multicolumn{2}{|l|}{ DES } & \multicolumn{3}{|c|}{ Odds Ratio } & \multirow{2}{*}{\multicolumn{5}{|c|}{$\begin{array}{c}\text { Odds Ratio } \\
\text { M-H, Random, } 95 \% \mathrm{Cl}\end{array}$}} \\
\hline & Events & Total & Events & Total & Weight & M-H, Random, $95 \% \mathrm{Cl}$ & Year & & & & & \\
\hline Costopoulas et al & 3 & 92 & 6 & 92 & $15.3 \%$ & $0.48[0.12,1.99]$ & 2014 & &. & & & \\
\hline Matesini et al & 1 & 35 & 2 & 28 & $5.1 \%$ & $0.38[0.03,4.45]$ & 2014 & 4 & & & & \\
\hline Sato et al & 9 & 96 & 8 & 96 & $30.8 \%$ & $1.14[0.42,3.09]$ & 2014 & & & & & \\
\hline BVS EXAMINATION & 5 & 290 & 4 & 290 & $17.5 \%$ & $1.25[0.33,4.72]$ & 2014 & & & - & & \\
\hline Cortese et al & 5 & 122 & 23 & 441 & $31.4 \%$ & $0.78[0.29,2.09]$ & 2015 & & & & & \\
\hline Total $(95 \% \mathrm{Cl})$ & & 635 & & 947 & $100.0 \%$ & $0.85[0.49,1.48]$ & & & & & & \\
\hline Total events & 23 & & 43 & & & & & & & & & \\
\hline $\begin{array}{l}\text { Heterogeneity: Tauz } \\
\text { Test for overall effect }\end{array}$ & $\begin{array}{l}0.00 ; \mathrm{Chi}^{\prime} \\
\mathrm{Z}=0.57\end{array}$ & $\begin{array}{l}z=1.71 \\
P=0.5\end{array}$ & df $=4(f$ & $P=0.79$ & 9); $P^{2}=0 \%$ & & & $\begin{array}{ll}\vdash & 1 \\
0.1 & 0.2\end{array}$ & $\begin{array}{c}0.5 \\
\text { Favours BVS }\end{array}$ & $\frac{2}{2}$ & 5 & 10 \\
\hline
\end{tabular}

Test for overall effect using Fixed Effect: $\mathrm{Z}=0.61(\mathrm{P}=0.54)$

C

\begin{tabular}{|c|c|c|c|c|c|c|c|c|c|c|c|}
\hline \multirow[b]{2}{*}{ Study or Subgroup } & \multicolumn{2}{|c|}{ BVS } & \multicolumn{2}{|c|}{ DES } & \multicolumn{3}{|c|}{ Odds Ratio } & \multirow{2}{*}{\multicolumn{4}{|c|}{$\begin{array}{c}\text { Odds Ratio } \\
\text { M-H, Random, } 95 \% \mathrm{Cl}\end{array}$}} \\
\hline & Events & Total & Events & Total & Weight & M-H, Random, $95 \% \mathrm{Cl}$ & Year & & & & \\
\hline BVS EXAMINATION & 6 & 290 & 4 & 290 & $20.0 \%$ & $1.51[0.42,5.41]$ & 2014 & & & T $-2=$ & \\
\hline Matesini et al & 1 & 35 & 0 & 38 & $3.1 \%$ & $3.35[0.13,84.92]$ & 2014 & & & & \\
\hline Costopoulos et al & 8 & 92 & 8 & 92 & $31.0 \%$ & $1.00[0.36,2.79]$ & 2014 & & & & \\
\hline Gori et al & 6 & 150 & 4 & 103 & $19.6 \%$ & $1.03[0.28,3.75]$ & 2014 & & & & \\
\hline Cortese et al & 5 & 122 & 9 & 441 & $26.3 \%$ & $2.05[0.67,6.24]$ & 2015 & & & & \\
\hline Total $(95 \% \mathrm{Cl})$ & & 689 & & 964 & $100.0 \%$ & $1.37[0.77,2.43]$ & & & & & \\
\hline Total events & 26 & & 25 & & & & & & & & \\
\hline $\begin{array}{l}\text { Heterogeneity: Tauz } \\
\text { Test for overall effect }\end{array}$ & $\begin{array}{l}0.00 ; \mathrm{Ch} \\
Z=1.08\end{array}$ & $\begin{array}{l}P^{2}=1.3 \\
P=0\end{array}$ & $\begin{array}{l}\text { 7. } d f=4 \\
\text { e) }\end{array}$ & $P=0.8$ & 5); $\left.\right|^{2}=0 x$ & & & $0.1 \quad 0.2$ & $\begin{array}{c}0.5 \\
\text { Favours BVS }\end{array}$ & $1 \frac{1}{2}$ & 10 \\
\hline
\end{tabular}

Test for overall effect using Fixed Effect: $\mathrm{Z}=1.05(\mathrm{P}=0.30)$

Figure 4 Death, target vessel revascularisation (TVR) and myocardial infarction (MI) with bioresorbable vascular scaffold (BVS) versus drug-eluting stent (DES) in registry studies in coronary artery disease. (A) Death, (B) TVR and (C) MI.

\begin{tabular}{|c|c|c|c|c|c|c|c|c|c|c|c|c|}
\hline \multirow{3}{*}{ Study or Subgroup } & \multicolumn{2}{|c|}{ BVS } & \multicolumn{2}{|c|}{ DES } & \multicolumn{3}{|c|}{ Odds Ratio } & \multirow{2}{*}{\multicolumn{4}{|c|}{$\begin{array}{c}\text { Odds Ratio } \\
\text { M-H, Random, } 95 \% \mathrm{Cl}\end{array}$}} & \\
\hline & Events & Total & Events & Total & Weight & M-H, Random, $95 \% \mathrm{Cl}$ & Year & & & & & \\
\hline & 4 & 150 & 3 & 103 & $23.2 \%$ & $0.91[0.20,4.17]$ & 2014 & & & & & \\
\hline Matesini et al & 0 & 35 & 2 & 38 & $5.7 \%$ & $0.21[0.01,4.44]$ & 2014 & $\longleftarrow$ & & & & \\
\hline BVS EXAMINATION & 7 & 290 & 4 & 290 & $34.8 \%$ & $1.77[0.51,6.11]$ & 2014 & & & F & & \\
\hline Costopoulas et al & 0 & 92 & 0 & 92 & & Not estimable & 2014 & & & & & \\
\hline Sato et al & 1 & 96 & 2 & 96 & $9.1 \%$ & $0.49[0.04,5.55]$ & 2014 & $\longleftarrow$ & & & & \\
\hline Cortese et al & 3 & 122 & 6 & 441 & $27.2 \%$ & $1.83[0.45,7.42]$ & 2015 & & & 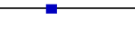 & & \\
\hline Total $(95 \% \mathrm{Cl})$ & & 785 & & 1060 & $100.0 \%$ & $1.21[0.58,2.51]$ & & & & & & \\
\hline Total events & 15 & & 17 & & & & & & & & & \\
\hline $\begin{array}{l}\text { Heterogeneity: Tauz } \\
\text { Test for overall effect }\end{array}$ & $\begin{array}{l}0.00 ; \mathrm{Ch} \\
\mathrm{Z}=0.50(\end{array}$ & $\begin{array}{l}z=2.66 \\
P=0.61\end{array}$ & $\begin{array}{l}3_{1}, \mathrm{df}=4(\mathrm{P} \\
\text { i) }\end{array}$ & $P=0.6$ & 2); $\left.\right|^{2}=0 \%$ & & & $\begin{array}{ll}1 & 1 \\
0.1 & 0.2\end{array}$ & $0^{1}{ }^{1}{ }^{1}$ & $\begin{array}{c}1 \\
\text { Favours DES }\end{array}$ & 5 & 10 \\
\hline
\end{tabular}

Test for overall effect using Fixed Effect: $\mathrm{Z}=0.35(\mathrm{P}=0.73$ )

$\mathrm{B}$

\begin{tabular}{|c|c|c|c|c|c|c|c|c|c|c|c|c|}
\hline \multirow[b]{2}{*}{ Study or Subgroup } & \multicolumn{2}{|c|}{ BVS } & \multicolumn{2}{|c|}{ DES } & \multicolumn{3}{|c|}{ Odds Ratio } & \multirow{2}{*}{\multicolumn{5}{|c|}{$\begin{array}{c}\text { Odds Ratio } \\
\text { M-H, Random, } 95 \% \mathrm{Cl}\end{array}$}} \\
\hline & Events & Total & Events & Total & Weight & M-H, Random, $95 \% \mathrm{Cl}$ & Year & & & & & \\
\hline Matesini et al & 0 & 35 & 2 & 38 & $6.7 \%$ & $0.21[0.01,4.44]$ & 2014 & 4 & & & & \\
\hline Gori et al & 3 & 150 & 2 & 103 & $19.3 \%$ & $1.03[0.17,6.28]$ & 2014 & & & & & \\
\hline Costopoulos et al & 0 & 92 & 0 & 92 & & Not estimable & 2014 & & & & & \\
\hline BVS EXAMINATION & 5 & 290 & 4 & 290 & $35.9 \%$ & $1.25[0.33,4.72]$ & 2014 & & & = & & \\
\hline Sato et al & 1 & 96 & 0 & 96 & $6.1 \%$ & $3.03[0.12,75.35]$ & 2014 & & & & & $\longrightarrow$ \\
\hline Cortese et al & 3 & 122 & 6 & 441 & $32.1 \%$ & $1.83[0.45,7.42]$ & 2015 & & & - & & \\
\hline Total $(95 \% \mathrm{Cl})$ & & 785 & & 1060 & $100.0 \%$ & $1.27[0.58,2.82]$ & & & & & & \\
\hline Total events & 12 & & 14 & & & & & & & & & \\
\hline \multicolumn{8}{|c|}{$\begin{array}{l}\text { Heterogeneity: Tau }=0.00 ; \mathrm{Ch}^{2}=1.97, \mathrm{df}=4(\mathrm{P}=0.74) ; \mathrm{I}^{2}=0 \% \\
\text { Test for overall effect: } Z=0.60(\mathrm{P}=0.55)\end{array}$} & $\begin{array}{ll} & 1 \\
0.1 & 0.2\end{array}$ & $\begin{array}{c}0.5 \\
\text { Favours BVS }\end{array}$ & $\begin{array}{c}\frac{1}{2} \\
\text { Favours DES }\end{array}$ & 5 & 10 \\
\hline
\end{tabular}

Test for overall effect using Fixed Effect: $\mathrm{Z}=0.45(\mathrm{P}=0.65)$

Figure 5 Any and definite stent thrombosis (ST) with bioresorbable vascular scaffold (BVS) versus drug-eluting stent (DES) in registry studies in coronary artery disease. (A) Any ST and (B) definite ST. 
$15.2 \pm 3.3 \mathrm{~atm}$. The use of intravascular imaging (OCT/ IVUS) guidance for vessel sizing and implant optimisation is recommended for BVS, but we found that this was performed in only a third of patients. In a recent multicentre study involving 1305 patients, the rate of ST with BVS was as high as $3 \%$, but this was significantly reduced (by $\sim 70 \%$ ) when optimal implantation strategy was employed. ${ }^{21}$ It is noteworthy that in registries that used high-pressure ( $\geq 20 \mathrm{~atm})$ BVS postdilation, and routine intravascular imaging guidance, there did not appear to be an increased risk of ST, ${ }^{11} 14^{16}$ and this probably shaped the findings of the meta-analysis of registry studies. Thus, a potential explanation for the observed increased risk of ST in the randomised trials may relate to suboptimal implantation techniques, rather than to the intrinsic properties of BVS. Furthermore, given the small number of patients included in this meta-analysis, it is also possible that the randomised trials were underpowered to detect a difference with respect to rare complications such as ST. Nevertheless, since ST is a rare but catastrophic complication, the available data provide cause for caution.

The Absorb BVS is a thick-strut scaffold, with the average strut thickness of $157 \mu \mathrm{m}$, which is almost equivalent to that of the first-generation Cipher stent (Cipher Bx Velocity $152.6 \mathrm{~mm}$, Cordis Corporation, Johnson \& Johnson, Warren, New Jersey, USA), and about double that of the second-generation Xience stent (Xience $\mathrm{V} 81.3 \mathrm{~mm}$ ). ${ }^{22}$ Indeed, the strut thickness is considered its main limitation, since it makes for a bulkier device which may prove challenging to pass across tortuous vessels or complex calcified lesions. Additionally, thick-strut, when compared with thin-strut, stents are associated with higher rates of angiographic restenosis and are considered to be thrombogenic. ${ }^{23} 24$ Thus, there may be increased neointimal thickness and thrombogenicity with BVS indicating perhaps the need to explore the requirement for long-term adjunct antithrombotic pharmacotherapy.

Despite the apparent difference in in-device LLL at median angiographic follow-up, this was not reflected in any difference in overall revascularisation rates for BVS versus DES at 1 year. Whether the observed greater loss in BVS lumen area beyond the first year after implantation translates into worse clinical outcomes is unknown. PCI techniques continue to rapidly evolve with improvements in stent platforms and adjunctive antiplatelet and antithrombotic therapy. With longer planned follow-up, the ABSORB II ${ }^{25}$ ABSORB IV (NCT02173379) and AIDA $^{26}$ trials will provide valuable insight into the longterm outcomes of BVS, particularly outcomes related to late and very late ST and lumen area.

In the studies included in this meta-analysis, the majority of patients were stable and had simple de novo lesions. Randomised trials included a higher percentage of patients with diabetes than did registries $(28.4 \%$ vs $18.2 \%$ ), but of note, the lesions were less complex (ACC/AHA lesion class was $\mathrm{B} 2 / \mathrm{C}$ in $66.2 \%$ of randomised trials vs $84.7 \%$ of registries). Despite the fact that virtually every study conducted in the era of stenting has shown higher event rates in patients with diabetes, ${ }^{27}$ there have been no head-to-head comparisons of outcomes comparing BVS in patients with and without diabetes. Muramatsu et $a l^{28}$ presented promising post hoc analyses 1 year after BVS implantation showing no difference in clinical outcomes, particularly in the incidence of subacute ST, with numerically low ST events in patients with diabetes.

Our results are in line with two previous meta-analyses by Cassese $e t a l^{29}$ and Lipinski $e t a l^{30}$ showing a higher incidence of ST and MI in the first year after BVS implantation. However, Stone $e t a l^{\beta 1}$ did not demonstrate the same pattern in a patient level, pooled meta-analysis of four randomised trials involving 3389 patients with CAD. Recently, a network meta-analysis of 147 trials showed that BVS had an increased risk of ST compared with contemporary DES. ${ }^{32}$ Our meta-analysis of data from randomised and registry studies suggest that this might indeed be related to inadequate implantation techniques, in particular to device underexpansion.

Our meta-analysis has several limitations. First, most studies used strict selection criteria and so, high-risk patients might not have been assessed, such as those with tortuous or calcified lesions. Second, all patients receiving BVS were treated with everolimus-eluting scaffold, in contrast to patients who received DES where the vast majority were treated with everolimus-eluting stent, and small numbers were treated with either biolimus-eluting stent $^{7}{ }^{11}$ or zotarolimus-eluting stent. ${ }^{16}$ It is however important to recognise that biolimus-eluting and zotarolimus-eluting stents had similar safety and efficacy as the everolimus-eluting stent in prior studies, ${ }^{33} 34$ and their inclusion is unlikely to confound the results of the meta-analysis. Third, three registry studies were propensity score matched-sample analyses, which did not directly compare the two devices. ${ }^{11} 13{ }^{14}$ However, a sensitivity analysis showed the same results when those studies were excluded. Fourth, data on adherence to dual antiplatelet therapy were unavailable in all but one registry. Furthermore, the studies included had some differences in design, definitions and follow-up periods. For randomised trials, the results of this meta-analysis are derived from study data and not from patient data. Availability of individual patient data could improve the reliability of the findings and permit more flexible analyses. Additionally, in one of the trials, 2-year results had been presented but not published. ${ }^{9}$ Finally, the randomised trials included were either single-blind or open-label with a potential for high performance bias.

Whether the higher risk of subacute ST observed with BVS reflects suboptimal implantation technique, thickstrut scaffold and/or lesion selection remains to be determined. Data from large observational studies are still needed, to confirm that BVS can deliver the same results as DES with the appropriate implantation techniques. Adequately powered randomised trials of BVS 
using careful lesion preparation and device optimisation are needed, with angiographic follow-up to assess clinical outcomes. Until then, caution must be exercised and when using BVS, every effort made to select suitable patients, with careful lesion preparation, with intervention guided by intravascular optimisation to embed the struts deeply in the vessel wall to avoid malapposition and to promote rapid endothelialisation.

\section{Author affiliations}

${ }^{1}$ Department of Cardiology, East and North Hertfordshire NHS Trust, Hertfordshire, UK

${ }^{2}$ Postgraduate Medical School, University of Hertfordshire, Hertfordshire, UK

${ }^{3}$ National Heart \& Lung Institute, Imperial College, London, UK

${ }^{4}$ St George's, University of London, London, UK

Acknowledgements The authors thank Ashraf Nabhan, MD, and David Wellsted, $\mathrm{PhD}$, for analytical support.

Contributors MF and NS did the literature search. All the authors analysed the data, interpreted the findings, drafted the manuscript and approved the manuscript submitted.

Competing interests None declared.

Provenance and peer review Not commissioned; externally peer reviewed.

Data sharing statement No additional data are available.

Open Access This is an Open Access article distributed in accordance with the Creative Commons Attribution Non Commercial (CC BY-NC 4.0) license, which permits others to distribute, remix, adapt, build upon this work noncommercially, and license their derivative works on different terms, provided the original work is properly cited and the use is non-commercial. See: http:// creativecommons.org/licenses/by-nc/4.0/

\section{REFERENCES}

1. Iqbal J, Sumaya W, Tatman V, et al. Incidence and predictors of stent thrombosis: a single-centre study of 5,833 consecutive patients undergoing coronary artery stenting. Eurolntervention 2013;9:62-9.

2. Kraak RP, Hassell ME, Grundeken MJ, et al. Initial experience and clinical evaluation of the Absorb bioresorbable vascular scaffold (BVS) in real-world practice: the AMC Single Centre Real World PCI Registry. Eurolntervention 2015;10:1160-8.

3. Capodanno D, Gori T, Nef H, et al. Percutaneous coronary intervention with everolimus-eluting bioresorbable vascular scaffolds in routine clinical practice: early and midterm outcomes from the European multicentre GHOST-EU registry. Eurolntervention 2015;10:1144-53.

4. Kočka $\mathrm{V}$, Malý M, Toušek $\mathrm{P}$, et al. Bioresorbable vascular scaffolds in acute ST-segment elevation myocardial infarction: a prospective multicentre study 'Prague 19'. Eur Heart J 2014;35:787-94.

5. Sabaté $M$, Windecker S, Iñiguez $A$, et al. Everolimus-eluting bioresorbable stent vs. durable polymer everolimus-eluting metallic stent in patients with ST-segment elevation myocardial infarction: results of the randomized ABSORB ST-segment elevation myocardial infarction-TROFI II trial. Eur Heart J 2016;37:229-40.

6. Gao R, Yang Y, Han Y, et al. Bioresorbable vascular scaffolds versus metallic stents in patients with coronary artery disease: ABSORB China trial. J Am Coll Cardiol 2015;66:2298-309.

7. Puricel S, Arroyo D, Corpataux N, et al. Comparison of everolimusand biolimus-eluting coronary stents with everolimus-eluting bioresorbable vascular scaffolds. J Am Coll Cardiol 2015:65:791-801.

8. Kimura T, Kozuma $\mathrm{K}$, Tanabe $\mathrm{K}$, et al. A randomized trial evaluating everolimus-eluting Absorb bioresorbable scaffolds vs. everolimus-eluting metallic stents in patients with coronary artery disease: ABSORB Japan. Eur Heart J 2015;36:3332-42.

9. Chevalier B, Serruys P. The 2-year clinical outcomes of the ABSORB II trial: first randomized comparison between the absorb everolimus eluting bioresorbable vascular scaffold and the XIENCE everolimus eluting stent. Transcatheter Cardiovascular Therapeutics; San Francisco, CA, USA, 2015.
10. Ellis SG, Kereiakes DJ, Metzger DC, et al. Everolimus-eluting bioresorbable scaffolds for coronary artery disease. N Engl J Med 2015;373:1905-15.

11. Sato K, Latib A, Panoulas VF, et al. Procedural feasibility and clinical outcomes in propensity-matched patients treated with bioresorbable scaffolds vs new-generation drug-eluting stents. Can J Cardiol 2015;31:328-34.

12. Cortese B, lelasi A, Romagnoli E, et al. Clinical comparison with short-term follow-up of bioresorbable vascular scaffold versus everolimus-eluting stent in primary percutaneous coronary interventions. Am J Cardiol 2015;116:705-10.

13. Brugaletta S, Gori T, Low AF, et al. Absorb bioresorbable vascular scaffold versus everolimus-eluting metallic stent in ST-segment elevation myocardial infarction: 1-year results of a propensity score matching comparison: the BVS-EXAMINATION Study (bioresorbable vascular scaffold-a clinical evaluation of everolimus eluting coronary stents in the treatment of patients with ST-segment elevation myocardial infarction). JACC Cardiovasc Interv 2015;8(Pt B):189-97.

14. Costopoulos $C$, Latib A, Naganuma T, et al. Comparison of early clinical outcomes between ABSORB bioresorbable vascular scaffold and everolimus-eluting stent implantation in a real-world population. Catheter Cardiovasc Interv 2015;85:E10-15

15. Gori T, Schulz E, Hink U, et al. Early outcome after implantation of Absorb bioresorbable drug-eluting scaffolds in patients with acute coronary syndromes. Eurolntervention 2014;9:1036-41.

16. Mattesini A, Secco GG, Dall'Ara G, et al. ABSORB biodegradable stents versus second-generation metal stents: a comparison study of 100 complex lesions treated under OCT guidance. JACC CardiovasC Interv 2014:7:741-50.

17. Moher $\mathrm{D}$, Liberati $\mathrm{A}$, Tetzlaff $\mathrm{J}$, et al. Preferred reporting items for systematic reviews and meta-analyses: the PRISMA Statement. Open Med 2009;3:e123-30.

18. Stroup DF, Berlin JA, Morton SC, et al. Meta-analysis of observational studies in epidemiology: a proposal for reporting Meta-analysis Of Observational Studies in Epidemiology (MOOSE) group. JAMA 2000;283:2008-12.

19. Cutlip DE, Windecker S, Mehran R, et al. Clinical end points in coronary stent trials: a case for standardized definitions. Circulation 2007;115:2344-51.

20. DerSimonian R, Laird N. Meta-analysis in clinical trials. Control Clin Trials 1986;7:177-88.

21. Puricel S, Cuculi F, Weissner M, et al. Bioresorbable coronary scaffold thrombosis: multicenter comprehensive analysis of clinical presentation, mechanisms, and predictors. J Am Coll Cardiol 2016;67:921-31.

22. Kawamoto H, Jabbour RJ, Tanaka A, et al. The bioresorbable scaffold: will oversizing affect outcomes? JACC Cardiovasc Interv 2016:9:299-300.

23. Kolandaivelu K, Swaminathan R, Gibson WJ, et al. Stent thrombogenicity early in high-risk interventional settings is driven by stent design and deployment and protected by polymer-drug coatings. Circulation 2011;123:1400-9.

24. Pache J, Kastrati A, Mehilli J, et al. Intracoronary stenting and angiographic results: strut thickness effect on restenosis outcome (ISAR-STEREO-2) trial. J Am Coll Cardiol 2003:41:1283-8.

25. Diletti R, Serruys PW, Farooq V, et al. ABSORB II randomized controlled trial: a clinical evaluation to compare the safety, efficacy, and performance of the Absorb everolimus-eluting bioresorbable vascular scaffold system against the XIENCE everolimus-eluting coronary stent system in the treatment of subjects with ischemic heart disease caused by de novo native coronary artery lesions: rationale and study design. Am Heart $J$ 2012;164:654-63.

26. Woudstra P, Grundeken MJ, Kraak RP, et al. Amsterdam Investigator-initiateD Absorb strategy all-comers trial (AIDA trial): a clinical evaluation comparing the efficacy and performance of ABSORB everolimus-eluting bioresorbable vascular scaffold strategy vs the XIENCE family (XIENCE PRIME or XIENCE Xpedition) everolimus-eluting coronary stent strategy in the treatment of coronary lesions in consecutive all-comers: rationale and study design. Am Heart J 2014;167:133-40.

27. Stone GW, Kedhi E, Kereiakes DJ, et al. Differential clinical responses to everolimus-eluting and Paclitaxel-eluting coronary stents in patients with and without diabetes mellitus. Circulation 2011:124:893-900.

28. Muramatsu T, Onuma Y, van Geuns RJ, et al. 1-year clinical outcomes of diabetic patients treated with everolimus-eluting bioresorbable vascular scaffolds: a pooled analysis of the ABSORB and the SPIRIT trials. JACC Cardiovasc Interv 2014;7:482-93.

29. Cassese S, Byrne RA, Ndrepepa G, et al. Everolimus-eluting bioresorbable vascular scaffolds versus everolimus-eluting metallic 
stents: a meta-analysis of randomised controlled trials. Lancet 2016;387:537-44.

30. Lipinski MJ, Escarcega RO, Baker NC, et al. Scaffold thrombosis after percutaneous coronary intervention with ABSORB bioresorbable vascular scaffold: a systematic review and meta-analysis. JACC Cardiovasc Interv 2016;9:12-24.

31. Stone GW, Gao R, Kimura T, et al. 1-year outcomes with the Absorb bioresorbable scaffold in patients with coronary artery disease: a patient-level, pooled meta-analysis. Lancet 2016;387:1277-89.
32. Kang SH, Chae IH, Park JJ, et al. Stent thrombosis with drug-eluting stents and bioresorbable scaffolds: evidence from a network meta-analysis of 147 trials. JACC Cardiovasc Interv 2016;9:1203-12.

33. Iqbal J, Serruys PW, Silber S, et al. Comparison of zotarolimus- and everolimus-eluting coronary stents: final 5-year report of the RESOLUTE all-comers trial. Circ Cardiovasc Interv 2015;8:e002230.

34. Natsuaki M, Kozuma K, Morimoto T, et al. Biodegradable polymer biolimus-eluting stent versus durable polymer everolimus-eluting stent: a randomized, controlled, noninferiority trial. J Am Coll Cardiol 2013;62:181-90. 